\title{
A Metacognição como Estratégia Reguladora da Aprendizagem
}

\author{
Metacognition as Regulatory Strategy of Learning
}

\author{
Graciela Inchausti de Jou ${ }^{a^{*}} \&$ Tania Mara Sperb ${ }^{b}$ \\ a Pontifícia Universidade Católica do Rio Grande do Sul, Porto Alegre, Brasil \\ ${ }^{b}$ Universidade Federal do Rio Grande do Sul, Porto Alegre, Brasil
}

\begin{abstract}
Resumo
A Psicologia Cognitiva através de seu enfoque do Processamento de Informação postula que a mente é um sistema cognitivo, que habilita o ser humano a interagir no seu meio. Este sistema, por sua vez, tem a capacidade de se monitorar e auto-regular, potencializando o próprio sistema. Essa capacidade foi definida como metacognição. O presente trabalho tem como finalidade, primeiramente, analisar o conceito de metacognição, tentando capturar sua essência e funcionalidade como processo cognitivo; em segundo lugar, mostrar e discutir os modelos expostos por diferentes autores da área e, por último, relatar pesquisas que mostram a metacognição como fator determinante na aprendizagem instrucional.

Palavras-chave: Metacognição; cognição; processamento de informação.

Abstract

Within Cognitive Psychology the Information Processing approach sees the mind as a cognitive system that allows the human being to interact in his/her environment. This system is capable of monitoring and selfregulating itself which, in turn, empowers the system all together. This capability has been defined as metacognition. The first aim of this study is to analyze the concept of metacognition, trying to capture its essence and functionality as a cognitive process. The second aim is to show and discuss the models provided by the different authors in the area. The final goal is to report a number of investigations that shows metacognition as a determinant factor for the instructional learning.

Keywords: Metacognition; cognition; information processing.
\end{abstract}

Imagine como seria nossa vida se não tivéssemos consciência de nossos próprios pensamentos. Como poderíamos planejar nossas ações e corrigi-las quando estas não ocorrem como esperado? Como poderíamos monitorar nossos comportamentos e adequá-los frente a cada exigência com a qual nos deparamos? Como poderíamos escolher a maneira mais adequada de estudar ao longo de nossa vida acadêmica? Podemos fazer tudo isto a cada instante devido à capacidade de nosso pensamento de pensar-se a si mesmo. A compreensão que as pessoas têm de seu próprio processamento cognitivo é denominada pela Psicologia Cognitiva de metacognição. Este campo de estudo começou a despontar nos anos 70 e intensificou-se nos anos 80 e 90.

Segundo Seminerio, Anselme e Chahon (1999), a metacognição como objeto de pesquisa abre um novo campo para as ciências cognitivas, provocando uma mudança de paradigma como também apontaram Nelson e Narens (1996).

Este artigo tem como finalidade, primeiramente, analisar o conceito de metacognição, tentando capturar sua essência e funcionalidade como processo cognitivo; em

\footnotetext{
Este trabalho faz parte de Tese de Doutorado da primeir autora sob orientação da segunda autora no Departamento de Psicologia do Desenvolvimento da Pós-Graduação de Faculdade de Psicologia da Universidade Federal do Rio Grande do Sul - UFRGS

* Endereço para correspondência: Rua Fernando Carneiro, 269, Três Figueiras, Porto Alegre, RS, 91330-100. Fone 3328 4134; Fax: 33289067. E-mail: grajou@terra.com.br; gjou@pucrs.br
}

segundo lugar, mostrar e discutir os modelos expostos por diferentes autores da área e, por último, relatar pesquisas que mostram a metacognição como fator determinante na aprendizagem instrucional.

\section{O Conceito de Metacognição}

A metacognição é a capacidade do ser humano de monitorar e auto-regular os processos cognitivos (Flavell, 1987; Nelson \& Narens, 1996; Sternberg, 2000). A essência do processo metacognitivo parece estar no próprio conceito de self, ou seja, na capacidade do ser humano de ter consciência de seus atos e pensamentos. Mas, o que é consciência?

Na língua portuguesa, utiliza-se a palavra consciência com múltiplos significados: para referir-se à vigília (estar consciente), à função superegóica (princípios morais que regem o indivíduo) e ao self, o qual remete à percepção do indivíduo de sua própria existência. O self se relacionaria ao que Damasio (1996) chamou de consciência ampliada, referindo-se à capacidade do indivíduo de ter consciência de quantidades enormes de entidades e eventos subjetivos, gerando uma perspectiva individual.

Por sua vez, Marx e Silva et al. (2004), enfatizando o caráter evolutivo da consciência, assinalam que o pensar sobre o próprio pensar, ou pensamento de segunda ordem, permitiu ao ser humano observar e corrigir seus pensamentos e suas ações, desenvolvendo estratégias cada vez mais sofisticadas para interagir com o meio e, portanto, para garantir sua sobrevivência. 
Com a finalidade de explicar essas funções metacognitivas, surgiram os modelos metacognitivos, explicitando a função auto-regulatória do sistema cognitivo.

\section{Modelos Metacognitivos}

Flavell e Wellman (1977) foram os primeiros autores a considerar a metacognição como uma área específica de pesquisa. Tulving (1996), no entanto, cita a tese de Hart (1965), sobre feeling of knowing (sentimento de saber), como a primeira tentativa de diferenciar cognição de metacognição, ou seja, de diferenciar cognição da cognição além da cognição. O sentimento de saber é, então, produto da função metacognitiva.

Inicialmente, os trabalhos sobre metacognição detiveram-se apenas no conhecimento que os indivíduos tinham sobre sua cognição, a exemplo do que sabiam sobre sua memória (metamemória) e do que sabiam sobre sua atenção, ou metatenção (Flavell \& Wellman, 1977). Definiuse, então, metacognição como a cognição sobre a cognição, ou como o processo mediante o qual o indivíduo realiza operações cognitivas, além de acompanhá-las enquanto elas acontecem (Flavell, 1987). Posteriormente, nas últimas décadas, novos conceitos foram sendo incorporados ao estudo de metacognição. Por exemplo, o enfoque do Processamento de Informação, proposto pela Psicologia Cognitiva, considera que o sistema cognitivo é provido de um subsistema de controle que tem a finalidade de monitorar, planejar e regular seus processos. Esse controle metacognitivo, na maioria das vezes, e, especialmente em crianças pequenas, acontece com pouca participação consciente. Entretanto, à medida que os processos cognitivos são mais exigidos por situações de vida mais complexas, os processos metacognitivos tornam-se mais conscientes, sendo a metacognição definida, então, como a capacidade de refletir conscientemente sobre os próprios processos cognitivos e metacognitivos (Brown, 1997; Leffa, 1996).

Yussen (1985), numa revisão comparativa dos modelos metacognitivos, indica, além do paradigma de Processamento de Informação, outros como o cognitivo-estrutural, o cognitivo-comportamental e o psicométrico, como aqueles que fizeram referência ao conceito de metacognição. Para o autor, os pesquisadores que trabalham com o paradigma do Processamento de Informação, como Siegler, Klahr, Sternberg e Trabasso, preocupam-se em descrever modelos de controle, envolvendo mecanismos de monitoramento e auto-regulação. Como conseqüência, também são descritos modelos de treinamento em estratégias e generalização. No que concerne ao paradigma cognitivo-estrutural, ao qual estariam ligados Piaget e Feldman, segundo Yussen (1985), a estrutura do conhecimento seria descrita, enfatizando-se as seqüências de mudança estrutural e elaborando-se modelos de relação entre mudança estrutural do conhecimento metacognitivo e outros conhecimentos. O paradigma cognitivo-comportamental, por sua vez, teria como representantes Bandura, Nischel, Rosenthal e Zimmerman (Yussen, 1985). Segundo esta orientação, a metacognição estaria integrada ao repertório de acontecimentos simbólicos que medeiam a aprendizagem. Descreve-se como a metacognição é modelada e relaciona-se esta modelagem com as mudanças de conduta. Já com relação ao paradigma psicométrico, o autor coloca que a psicometria tenta identificar fatores metacognitivos nos desempenhos dos testes. Castell-Horn e Guilford seriam, de acordo com Yussen, os representantes mais importantes desta orientação.

Essa categorização de Yussen (1985) objetiva rastrear alguns indicadores da metacognição nos diferentes paradigmas cognitivos. Brown (1997), no entanto, reconhece que as raízes da metacognição encontram-se exclusivamente no paradigma do Processamento de Informação, mais especificamente, na descrição dos mecanismos executivos do sistema cognitivo para aprendizagem, por meio do desenvolvimento da auto-regulação, e na instrução, por meio do desenvolvimento da heterorregulação.

De uma forma mais ampla, Mayor, Suengas e González Marqués (1995) entendem que o conceito de metacogni-ção pode ser encontrado também nos estudos sobre consciência reflexiva, teoria da mente, modelos mentais, processamento voluntário ou automático, al terações neurológicas, auto-eficácia, auto-estima e retroalimentação, já que todos esses assuntos implicam no conhecimento consciente da própria mente.

Essa compreensão mais ampla também foi apontada por Flavell (1987). O autor, ao definir metacognição como o conhecimento que o sujeito tem sobre os eventos cognitivos, caracteriza a magnitude do campo metacognitivo. Ao mesmo tempo, entretanto, alerta para o perigo que essa amplidão traz para o conceito de metacognição, uma vez que ela pode referir-se a todos os fenômenos psicológicos. Por exemplo, se alguém tem o conhecimento sobre as motivações ou emoções próprias, este conhecimento poderia ser considerado metacognitivo, assim como qualquer tipo de conhecimento que o sujeito tenha sobre si mesmo, seja de uma atividade motriz ou de uma atividade cognitiva ou, ainda, de um sentimento. Portanto, segundo Flavell (1987), é necessário especificar o domínio da metacognição. Com essa finalidade, o autor diferencia os tipos de conhecimento necessários na atividade metacognitiva. Ele destaca, por um lado, o conhecimento metacognitivo e a experiência metacognitiva e, por outro, os objetivos e as ações cognitivas.

A Figura 1 expõe o modelo de metacognição de Flavell (1987).

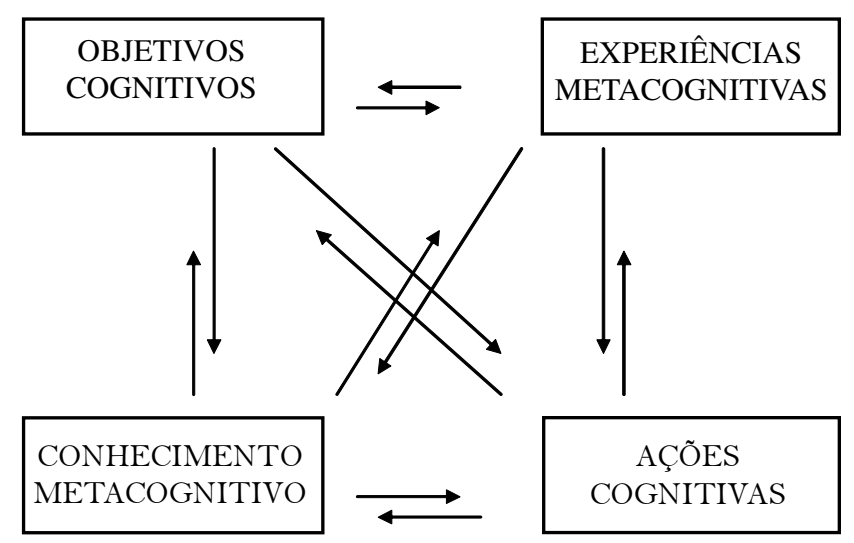

Figura 1. Modelo de metacognição de Flavell (1979, 1987), adaptado por Mayor et al. (1995, p. 32). 
O conhecimento metacognitivo refere-se ao conhecimento adquirido pelo indivíduo com relação ao todo cognitivo - sua mente e suas características psicológicas -, e as experiências metacognitivas referem-se à consciência das experiências cognitivas e afetivas que acompanham cada empreendimento cognitivo. Os objetivos cognitivos, por sua vez, referem-se às metas a serem alcançadas em cada envolvimento cognitivo e as ações cognitivas referem-se às realizações para atingir tais metas. Especificando ainda mais o modelo, o autor divide o conhecimento metacognitivo em três subcategorias de conhecimento que compõem: (a) variáveis da pessoa; (b) variáveis da tarefa; e (c) variáveis da estratégia.

O conhecimento das variáveis pessoais refere-se ao conhecimento que o sujeito adquiriu sobre os aspectos da cognição humana (universais), sobre as habilidades e motivações dos outros (interindividuais) e sobre as habilidades e motivações próprias (intra-individuais). Freqüentemente, é possível escutar comentários de estudantes como "Se eu tivesse prestado atenção na sala de aula, agora lembraria a matéria", ou "Se não tivesse ficado nervoso no vestibular teria lembrado a maioria das respostas", ou seja, existe todo um conhecimento adquirido pela experiência, concernente ao próprio funcionamento cognitivo e afetivo.

O conhecimento das variáveis da tarefa refere-se ao conhecimento que o indivíduo adquiriu sobre como lidar com as informações. Por exemplo, as pessoas sabem que informações familiares exigem menos esforço atencional que informações totalmente novas, assim como sabem que é mais fácil lembrar a idéia central de uma história do que as palavras exatas utilizadas. Esse conhecimento permite, então, avaliar as diferenças entre as tarefas e organizar-se para o cumprimento destas.

Com relação às variáveis de estratégia, Flavell (1987) ainda faz uma distinção entre estratégias cognitivas e metacognitivas. As primeiras dizem respeito ao resultado de uma tarefa e as segundas, à eficiência deste resultado. Por exemplo, para resolver uma adição soma-se um número a outro. Essa é uma estratégia cognitiva. Repetir a operação várias vezes para ter confiança de que a estratégia cognitiva utilizada levou ao sucesso é uma estratégia metacognitiva.

Quanto à experiência metacognitiva, Flavell (1987) esclarece que a mesma está relacionada à experiência subjetiva do indivíduo no que diz respeito a um determinado evento cognitivo. Assim, ter o sentimento de que não se está entendendo o que foi lido, ou sentir a conhecida sensação de que uma palavra está na ponta da língua, ou ter o sentimento de que se sabe algo (feeling of knowing) seriam experiências metacognitivas. Segundo o autor, várias pesquisas indicam que crianças pequenas podem ter tais experiências, mas não sabem interpretá-las. Com o desenvolvimento, as crianças tomam consciência do que estas experiências significam e quais seriam suas implicações para o comportamento.

O fato de que o processo metacognitivo é, por sua vez, também um processo cognitivo faz com que muitas vezes seja difícil delimitar as fronteiras entre o cognitivo e o metacognitivo. Para tal, segundo Flavell (1987), seria importante criar modelos detalhados de Processamento de Informação para os vários aspectos da metacognição. Esses modelos, segundo o autor, levantariam questões tais como que informação acerca dos processos cognitivos seria necessária para monitorar e regular esses processos e/ou quais seriam os indicadores cognitivos a serem observados.

Dentro da visão de Processamento de Informação, Flavell (1987) sugere que um indicador que poderia ser utilizado, por exemplo, na leitura, seria o da velocidade de processamento. Um sistema metacognitivo que monitora uma repentina mudança na velocidade da leitura permitiria ao sujeito durante o ato de leitura perceber que o material está se tornando difícil ou que outro tópico está solicitando uma maior atenção, podendo assim atuar para mudar a estratégia de leitura. O sistema estaria supervisionando, simultaneamente, tanto as variáveis da tarefa quanto as variáveis pessoais, permitindo alterar as estratégias utilizadas. Dessa maneira, o autor outorga ao processo metacognitivo uma capacidade auto-reguladora que age sobre o próprio sistema cognitivo.

O modelo de Flavell (1987) destaca, ainda, a possível interação entre o desenvolvimento dos processos metacognitivos e os processos de informação elementares, referindo-se à influência das limitações no conteúdo específico sobre a aquisição de algum tipo de metacognição. Isto é, o conhecimento metacognitivo específico de determinado domínio se desenvolveria só depois do sujeito ter suficiente conhecimento sobre esse domínio. E exemplifica, dizendo que um indivíduo não alfabetizado nunca poderia desenvolver habilidades metacognitivas para a leitura.

Na mesma linha de pensamento, Miller (1993) também chama a atenção para a relação metacognição e conhecimento específico. A autora define metacognição como um processo cognitivo que exige, para atuar, um determinado conhecimento. Por exemplo, a própria atividade de leitura permite a utilização de estratégias específicas. Essas estratégias, por sua vez, facilitam a recuperação e o desenvolvimento do conhecimento específico da leitura, criando novas associações e aumentando, conseqüentemente, a capacidade de leitura.

Levando em consideração, por um lado, os conhecimentos mais gerais e, por outro, os conhecimentos específicos, é possível, conforme Bruer (1995), pensar em dois níveis da atividade metacognitiva. Um mais geral que permite refletir, por exemplo, sobre resoluções de problemas do cotidiano e outro nível, mais específico, relacionado a resoluções de problemas de cada domínio.

O modelo inicial proposto por Flavell (1979) teve ao longo dos anos vários ajustes, incorporando conhecimentos tanto teóricos, provenientes do enfoque do Processamento de Informação, quanto empíricos, provenientes de resultados de diferentes pesquisas, como, por exemplo, daquelas com aprendizes eficientes. Essas pesquisas indicaram que os aprendizes eficientes tinham uma maior habilidade metacognitiva (Ertmer \& Newby, 1996). 
Recentemente, tem se destacado na literatura o modelo metacognitivo proposto por Nelson e Narens (1996), que enfatiza o fluxo de informação entre dois níveis, meta e objeto. Este sistema, como idealizado pelos autores, está exposto na Figura 2.

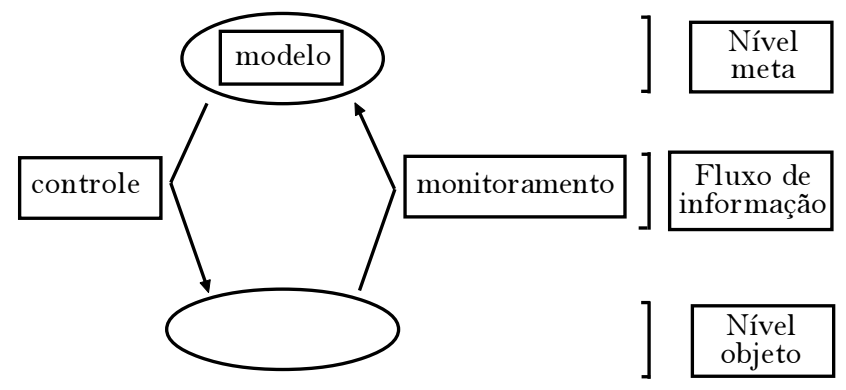

Figura 2. Modelo de Nelson e Narens, (1996, p. 11).

Nelson e Narens (1996) focalizam os processos de monitoração e auto-regulação da habilidade metacognitiva e enfatizam o estudo dos mecanismos reflexivos envolvidos nas respostas obtidas em situações do cotidiano. Os autores propõem que um sistema metacognitivo eficiente, além de monitorar ou refletir sobre o processamento, precisa controlá-lo.

Segundo este modelo, o sistema metacognitivo tem dois níveis - nível meta e nível objeto - e duas relações de fluxo de informação entre os níveis - controle e monitoramento. Para os autores, há uma relação de hierarquia no sistema metacognitivo: o nível meta tem o poder de modificar o nível objeto em função das informações obtidas deste. Essa possibilidade do processo metacognitivo "saltar", como referem os autores, entre os níveis inter-relacionados é dada pela direção no fluxo da informação.

Para Nelson e Narens (1996), o nível objeto seria o nível de atuação cognitiva e o nível meta seria o nível de atuação metacognitiva. Assim, o sistema metacognitivo, segundo os autores, funcionaria da seguinte maneira: enquanto a cognição «salta» para o nível meta ocorre o monitoramento do nível objeto por meio da construção de um modelo. Neste caso, modelo, para os autores significa a representação mental da realidade desse momento cognitivo. Já, quando a cognição "salta” para o nível objeto ocorre o controle mediante a regulação do processo cognitivo. Ou seja, em função da informação vinda do nível meta (do modelo ou representação mental), a cognição no nível objeto tem condições de se auto-regular, atendendo às exigências da atividade cognitiva como um todo.

Concluindo, para que o monitoramento aconteça deve haver fluência da informação do nível objeto para o nível meta e, para que a auto-regulação aconteça, há de fluir informação do nível meta para o nível objeto.

Ao analisar os modelos citados, é possível concluir que, enquanto o modelo metacognitivo de Flavell (1987) enfatiza os componentes ou as estruturas que fazem parte do sistema metacognitivo, o modelo de Nelson e Narens (1996) focaliza o processo de fluxo de informação desse sistema. Assim, os dois modelos podem ser integrados dentro de uma visão mais global do funcionamento metacognitivo.

Esta possibilidade de integração dos modelos de Flavell (1987) e de Nelson e Narens (1996) é contemplada no exemplo de um estudante resolvendo um problema matemático. Ao começar a resolver um problema, o aluno lê as informações e faz uma primeira representação destas, elaborando uma representação mental com os dados do problema e os conhecimentos pertinentes ativados na memória. Dentre esses conhecimentos pertinentes, estariam os elementos cognitivos propostos por Flavell, ou seja, o conhecimento e a experiência metacognitivos, objetivos e as ações cognitivas. De posse desses dados, elabora-se um modelo de resolução de problema no nível meta. A partir do modelo, a cognição atua na resolução do problema intercambiando dados com o modelo, refazendo continuamente o modelo até a resolução final do problema. Desta forma, desde o primeiro momento, trabalha-se na resolução do problema mediante a elaboração de modelos, os quais podem ser monitorados e modificados ao longo do processo, por meio do sistema metacognitivo.

Sintetizando o exposto até agora, a metacognição não se caracteriza somente como conhecimento sobre cognição, mas é hoje entendida como uma fase de processamento de alto nível que é adquirida e desenvolvida pela experiência e pelo acúmulo do conhecimento específico. Em função desse processamento supra-ordenado, o indivíduo consegue monitorar, auto-regular e elaborar estratégias para potencializar sua cognição. Neste sentido, a metacognição tornou-se de grande importância para as propostas de instrução educacional que valorizam o uso de estratégias metacognitivas na aprendizagem, como mostram, por exemplo, no Brasil, os estudos, tanto na aprendizagem da matemática quanto da linguagem, realizados no Laboratório de Metacognição, criado em 1991 na Universidade Federal do Rio de Janeiro (UFRJ), pelo Prof. Dr. Franco Lo Presti Seminerio.

\section{Metacognição e Aprendizagem Instrucional}

Miller (1993) indica que uma das grandes mudanças no desenvolvimento, durante os anos escolares e na adolescência, é a aprendizagem de como maximizar o uso das capacidades cognitivas mediante as capacidades metacognitivas. Por exemplo, no estudo acadêmico, à medida que os alunos adquirem maior experiência, é possível fazer melhor uso do tempo de estudo, selecionando os tópicos relevantes e ignorando os irrelevantes. Brown (1978) também observou que, com o passar do tempo, os estudantes aumentam consideravelmente o controle das estratégias que utilizam e de outros processos cognitivos. As crianças, para a autora, já começam a harmonizar os processos cognitivos, aumentando o controle sobre o conjunto formado por eles como se aprendessem a conduzir uma grande orquestra.

Blakey e Spence (2000), numa especificação mais rigorosa do que seriam atividades metacognitivas, indicam três estratégias metacognitivas básicas: (a) saber relacionar 
novas informações às já existentes, (b) saber selecionar estratégias de pensamento com um propósito e (c) saber planejar, monitorar e avaliar os processos de pensamento. Nessa perspectiva, segundo os autores, alunos que soubessem utilizar com eficiência essas estratégias metacognitivas seriam aprendizes eficientes.

Ertmer e Newby (1996) entendem que a reflexão, nos processos de aprendizagem, é o ingrediente essencial para o desenvolvimento de aprendizes eficientes. Esses autores estudaram como estes sujeitos utilizam o conhecimento que têm sobre si próprios como aprendizes, sobre as exigências das tarefas e sobre os métodos utilizados conscientemente para selecionar, controlar e monitorar as estratégias necessárias para alcançar uma aprendizagem eficiente. Baseados nesses estudos, Ertmer e Newby criaram um modelo de aprendizagem eficiente que mostra como o conhecimento metacognitivo das estratégias consideradas por eles cognitivas, motivacionais e ambientais, é traduzido em um controle auto-regulador dos processos de aprendizagem, por meio do pensamento reflexivo.

O modelo de Ertmer e Newby (1996) inclui os processos de planejar, controlar e refletir, destacando, sobretudo o caráter consciente desses processos. Na aprendizagem eficiente, o sujeito estaria consciente do conhecimento específico, das metas que tem que alcançar, das estratégias necessárias para alcançá-las, assim como do próprio processo no momento de seu acontecimento. Essa atividade on-line, como muitos autores a caracterizam utilizando a metáfora computacional, traz como conseqüência o processo auto-regulador. Os indivíduos, hábeis metacognitivamente, teriam a capacidade de incorporar e aplicar vários conhecimentos para aperfeiçoar o desempenho acadêmico, transformando-se em aprendizes eficientes. Seriam, por exemplo, capazes de saber o quê sabem (conhecimento declarativo), como utilizar o que sabem (conhecimento procedural) e por que, onde e quando utilizar o que sabem (conhecimento condicional, contextual), aplicando as estratégias relevantes ao objetivo da atividade cognitiva.

Já na década de 70, Flavell e Wellman (1977) destacaram níveis diferentes de aprendizagem. Para eles, o nível superior de aprendizagem relaciona-se às atividades metacognitivas e os aprendizes podem ser classificados desde o nível de principiantes até eficientes. A criança pequena, para estes autores, seria o aprendiz principiante universal.

A aprendizagem exige transformações e essas são relacionadas por Flavell e Wellman (1977) aos quatro níveis de funcionamento da atividade mental na aprendizagem. Há um primeiro nível, mais elementar, em que os conteúdos da memória organizam-se segundo regras de associação, mediante os processos básicos inatos. Nesse nível, acontece a aprendizagem dos condicionamentos e automatismos. Por exemplo, quando o bebê chora porque tem fome e recebe o alimento, o bebê aprende a associar seu choro ao recebimento do alimento. No segundo nível, seriam adicionados os conhecimentos declarativos/semânticos, organizados em esquemas mediante os processos básicos da estrutura cognitiva. A aquisição da linguagem seria o exemplo mais relevante desse nível. No terceiro nível estariam as estratégias e os métodos fracos e fortes utilizados voluntária e conscientemente por meio dos processos cognitivos superiores, por exemplo, ao relacionar os conceitos ou categorizá-los. Nesse nível, podem ser verbalizados conceitos, definições e suas relações, de forma mais reprodutiva ou de forma mais reconstruída, segundo os métodos fracos ou fortes utilizados na sua aprendizagem. O quarto nível, que seria o metacognitivo, envolveria o conhecimento, a consciência e o controle dos outros níveis. Trata-se da consciência do próprio sujeito como aprendiz ou processador de símbolos, como propõe o modelo do Processamento de Informação.

Esses níveis podem ser utilizados tanto para analisar as etapas de desenvolvimento do indivíduo, como nos exemplos citados, quanto para analisar as etapas de aprendizagem de um conhecimento novo. Dessa maneira, pode-se diferenciar um aluno que desenvolve habilidades mais eficientes de aprendizagem, isto é que reconstrói e opera com conceitos, daquele que está apenas repetindo ou reproduzindo conceitos.

Nessa linha de pensamento, Ribeiro (2003), propõe que a variável metacognitiva no estudo da aprendizagem permite introduzir conceitos como a auto-apreciação e o autocontrole cognitivos do aprendiz, assim como, trabalhar com diferenças pessoais e considerar a possibilidade da metacognição impulsionar o próprio desenvolvimento cognitivo.

De uma forma geral, pode-se dizer que a metacognição é uma conquista evolutiva do ser humano, em sua constante tarefa de adaptação, que se desenvolve para atender as exigências do ambiente. Utilizando o paradigma darwiniano, poderia dizer-se ainda, que os processos mentais tendem à complexidade e à automatização, permitindo atingir novos níveis de desenvolvimento mental.

\section{A Metacognição no Ambito Educacional}

Vários trabalhos empíricos têm mostrado a metacognição como um fator determinante na aprendizagem formal. Por exemplo, Klauer (1996) mostrou o monitoramento dos processos cognitivos envolvidos na resolução de tarefas em diferentes domínios escolares; já Vieira (1999), analisou a auto-instrução durante a resolução de problemas matemáticos, em professoras de ensino fundamental. A metacognição na alfabetização foi estudada por Sena de Paula (2000). Estudos sobre a metacognição aplicada à aprendizagem da matemática (Oliveira et al., 2000) e aplicada à compreensão da leitura (Boruchovitch \& Mercuri, 1999; Forest-Pressley \& Waller, 1984; Garner, 1987) revelaram a importância da relação do domínio específico com o desenvolvimento da capacidade metacognitiva desse domínio.

A importância do domínio específico nos estudos empíricos sobre metacognição pode ser apreciada na aprendizagem da leitura compreensiva. Segundo Leffa (1996), neste campo, a reflexão do leitor acerca de seu processo cognitivo de compreensão tem merecido a atenção dos 
investigadores. Para tal, tem se observado o uso que o sujeito faz de estratégias cognitivas e metacognitivas.

O principal procedimento utilizado nessas investigações é a análise de protocolo. Esta, aplicada à pesquisa da leitura, consiste essencialmente em uma entrevista realizada com o leitor e posterior análise detalhada das respostas. Solicita-se ao leitor que descreva seus processos de leitura e as estratégias que emprega para compreender o que lê.

De acordo com Leffa (1996), quatro conclusões principais emergiram dos resultados desse tipo de pesquisa. A primeira conclusão assinala que a atividade metacognitiva caracteriza-se como resultado do desenvolvimento. $\mathrm{O}$ autor diz que as crianças mais novas não são capazes de avaliar a sua própria compreensão, concordando com Miller (1993), quando afirma que a metacognição desenvolve-se com a idade. Uma segunda conclusão estabelece a correlação entre metacognição e proficiência em leitura, isto é, quanto mais intenso for o hábito de leitura do sujeito, maior será a sua capacidade para avaliar a sua própria compreensão e, conseqüentemente, para utilizar estratégias de leitura mais adequadas. Isso porque, segundo Flavell (1987), as estratégias metacognitivas específicas desenvolvem-se ao mesmo tempo em que aumenta a experiência na área em questão. A terceira conclusão indica que a instrução influencia o desenvolvimento metacognitivo, isto é, quando a criança é exposta a um programa sistemático de monitoramento de leitura compreensiva, sua habilidade de leitura melhora.

A última conclusão apontada por Leffa (1996) refere-se à importância do objetivo do leitor que determinará a eficácia de uma determinada estratégia de leitura. Por exemplo, leituras que exigem maior esforço para serem compreendidas induzem ao aparecimento de estratégias de maior comprometimento cognitivo, levando a uma compreensão mais profunda do texto. Essa conclusão relaciona-se a uma das premissas básicas da Psicologia Cognitiva, a de que no funcionamento cognitivo há uma estreita relação entre os objetivos e as estratégias empregadas. Quanto mais os objetivos cognitivos são difíceis, mais desenvolvidas devem ser as estratégias utilizadas para atingilos, exigindo processamentos de alto nível. De acordo com Stillings et al. (1995), aloca-se nos processos cognitivos de alto nível uma maior energia mental, ou seja, um maior esforço cognitivo. A leitura compreensiva é um desses processos de alto nível.

$\mathrm{Na}$ revisão da literatura sobre leitura compreensiva, constata-se que as variáveis que influenciam a compreensão de texto, como o conhecimento das superestruturas, a construção de macroestruturas e a utilização de ações cognitivas e metacognitivas, em maior ou menor grau, podem ser ensinadas de forma explícita e direta. Por exemplo, Vidal-Abarca (1990), em seu estudo, instruiu crianças de $5^{a}$ série a elaborar a idéia principal do texto e a tomar consciência do tipo de texto que estavam lendo, utilizando para esse fim textos expositivos. O procedimento empregado foi o de instrução direta, já que envolve a orientação explícita e sistemática do aluno durante realização da tarefa. Isso é feito através de exercícios específicos que têm a finalidade de aprimorar o desempenho. Nesse contexto, o aluno é ensinado a monitorar o andamento de sua atividade, recebendo um feedback direto e imediato do instrutor. Vidal-Abarca utilizou-se de um delineamento pré e pós-teste, com um grupo de controle de mesma idade e escolaridade. O pesquisador examinou nas leituras de diversos textos três tipos de medidas dependentes: (a) elaboração da idéia principal; (b) consciência da superestrutura (tipo de texto) e (c) recuperação do texto. Os resultados de seu trabalho mostraram que o grupo experimental saiu-se melhor do que o de controle nas três variáveis investigadas. O estudo também mostrou que a compreensão de texto está diretamente relacionada ao conhecimento do leitor acerca das estruturas textuais, ou seja, da superestrutura e da macroestrutura de um determinado texto.

Na mesma linha, Jou (2001) observou que as crianças que participaram de um programa de intervenção em leitura compreensiva aumentaram seu conhecimento sobre a estrutura do texto e sobre suas capacidades metacognitivas. No estudo, foram utilizados quatro tipos de medidas dependentes em um delineamento pré e pósteste. $\mathrm{O}$ primeiro tipo referiu-se à compreensão do texto, a qual foi avaliada mediante duas atividades. Uma em que os alunos escreviam o que se lembravam do texto e outra em que respondiam a um questionário de múltipla escolha sobre o texto lido. O segundo tipo de medidas envolveu a estrutura de texto. Para avaliar este conhecimento, foi solicitado aos alunos que identificassem as frases mais importantes, que escrevessem a idéia principal do texto e que indicassem o tipo de texto que tinham lido (se narrativo ou informativo). O terceiro tipo de medida compreendeu as estratégias cognitivas. Com a finalidade de avaliar este conhecimento, os alunos destacaram as estratégias utilizadas para leitura. O último tipo de medida referiu-se às estratégias metacognitivas, avaliadas por um questionário que investigava as reflexões feitas pelo aluno sobre sua leitura.

Depois do período de intervenção, no qual se utilizou o método de instrução direta (Pressley, Goodchild, Fleet, Zajchowski \& Evans, 1989; Vidal-Abarca, 1990), os alunos mostraram progresso no que concerne ao conhecimento da estrutura textual e à capacidade de refletir sobre a leitura, isto é, na metacognição, assim como na própria leitura compreensiva. Este fato permite supor que as variáveis trabalhadas ao longo da intervenção (estrutura textual e estratégias cognitivas e metacognitivas) provocaram um desempenho melhor dos alunos do grupo experimental na compreensão da leitura, quando comparados aos alunos dos grupos de controle.

Em outra linha de pesquisa, mas ainda relacionada à compreensão de leitura, pode ser citado o estudo de Boruchovitch (2001). A autora investigou as estratégias espontâneas de aprendizagem mencionadas por alunos de ensino fundamental e as relacionou com as variáveis: idade, série escolar, gênero e repetência. A amostra estava composta de 110 alunos, entre 8 e 16 anos de idade de terceira, quinta e sétima séries de uma escola selecionada por apresentar problemas de repetência. Os alunos per- 
tenciam a um nível socioeconômico menos favorecido. $\mathrm{O}$ instrumento utilizado foi traduzido e adaptado pela autora do Self-Regulated Learining Structured Interview de Zimmerman e Martinez-Pons (1986). Os resultados do estudo embora não mostrarem relação significativa entre as estratégias de compreensão de leitura e as variáveis: idade, gênero e série, mostraram o efeito de repetência, ou seja, os alunos repetentes apontaram menos uso de estratégias de compreensão de leitura.

Com relação à estratégia específica de leitura de fazer resumo, Thiede e Anderson (2003) examinaram a relação entre essa estratégia e eficiência no monitoramento da compreensão de leitura. Os estudantes da amostra foram divididos em dois grupos, um grupo experimental, orientado a resumir o conteúdo de texto lido por um dos colegas e um grupo controle que não fez resumo. Ainda o grupo experimental foi subdividido em um grupo que fazia o resumo imediatamente após a leitura do texto e outro grupo que o fazia uns dias depois. Posteriormente, foi aplicado um teste sobre o conteúdo do texto lido em ambos os grupos. Os resultados mostraram que o grupo que fez o resumo depois de um tempo, teve um desempenho superior comparado com o desempenho, tanto do grupo que não fez o resumo, quanto do grupo que o fez imediatamente. Os autores apontam para a importância do fator tempo na organização do conhecimento e na aprendizagem auto-regulada.

A aprendizagem das matemáticas é outra área específica onde é pesquisada a habilidade metacognitiva. O estudo de Chahon (1999), sobre a utilização sistemática da capacidade metacognitiva em uma aula de matemática, mostra a possibilidade de promover a compreensão de regras lógicas para resolução de frações. Os participantes foram crianças de $4^{\mathrm{a}}$ série do ensino fundamental com idades entre oito e treze anos. O autor selecionou dois grupos experimentas e dois grupos controle com 10 alunos cada grupo. O período de treinamento envolveu doze atividades ao longo de dez encontros onde as crianças foram estimuladas, através da metodologia de elaboração dirigida, a uma reflexão sobre as regras ou invariâncias necessárias para a compreensão dos conceitos fracionários.

Todavia com relação à aprendizagem da matemática, pode ser citado o estudo de Mevarech e Kramarski (2003). Este estudo longitudinal foi realizado com 122 alunos de oitava série de uma escola de Israel e o objetivo foi investigar dois aspectos: (a) o efeito do treinamento metacognitivo e do treinamento na resolução de problemas de álgebra nos alunos da amostra e (b) o efeito de longo prazo dos dois métodos no desempenho dos alunos no ano seguinte, quando estivessem cursando a nona série. Os resultados mostraram que os alunos que participaram do treinamento metacognitivo tiveram melhor desempenho que os que praticaram a resolução do problema, em ambos pós-testes, no imediato e no ano seguinte.

Com relação às matemáticas, observa-se o interesse também no estudo das habilidades metacognitivas do professor para ensinar estratégias de pensamento como mostram a pesquisa de Vieira (1999) e o estudo de Erktin
(2004). Este último desenvolveu um projeto no qual os professores de matemática foram introduzidos no debate sobre a possibilidade de ensinar estratégias de pensamento. Foram conduzidos três estudos com a finalidade de investigar o papel de metacognição no desempenho matemático e o efeito das habilidades metacognitivas do professor.

A metacognição relacionada à resolução de problemas é investigada, também, dentro da área instrucional. Rozencwajg (2003), por exemplo, selecionou 42 alunos de sétima série, com idades entre 12 e 13 anos, para investigar em que medida os níveis metacognitivos relacionavam-se com seus desempenhos na resolução de problemas de ciências na escola. Dois indicadores de metacognição foram estabelecidos pela autora: (a) o metaconhecimento acerca da aprendizagem em sala de aula e (b) o monitoramento metacognitivo da dificuldade do problema não acadêmico proposto na pesquisa. Os dois indicadores foram relacionados ao desempenho dos alunos em testes de QI e a estratégias de resolução de problemas de eletricidade. Os resultados mostraram que o metaconhecimento estava relacionado à inteligência cristalizada, enquanto o monitoramento metacognitivo aparecia associado à inteligência fluída. No entanto, ambos indicadores metacognitivos estavam for temente relacionados às estratégias de resolução de problemas científicos.

Além das pesquisas sobre metacognição em domínios específicos, como os citados acima, surgiram nos últimos anos pesquisas com novas propostas metodológicas para o estudo da metacognição. Nelson, Narens e Dunlosky (2004) e Kimball e Metcalfe (2003) investigaram o monitoramento metacognitivo, utilizando a técnica de julgamento da aprendizagem. Com tal finalidade, analisaram o efeito do julgamento na recuperação do material aprendido, concluindo que a recuperação dos conhecimentos aprendidos está intimamente relacionada ao julgamento que o indivíduo faz de sua aprendizagem.

Nesta mesma linha de investigação, Son (2004) pesquisou o controle que os indivíduos tinham quanto à utilização de estratégias para estudar um material que poderia ser examinado ou todo de uma só vez, maciçamente, ou de forma espaçada, em intervalos de tempo. A autora apresentou três predições para o estudo, que tinha como objetivo a aprendizagem de longa duração: (a) a predição de estudar de forma compacta sugere que as pessoas confiariam no estudo intensivo, feito em um só momento; (b) a de estudar em intervalos sugere que as pessoas escolheriam espaçar seus momentos de estudo e; (c) a predição metacognitiva sugere que as pessoas controlariam seus horários de estudo em função do jul gamento metacognitivo que fazem do material a ser aprendido.

Para testar estas predições, Son (2004) realizou um estudo no qual solicitou aos participantes estudar e fazer o julgamento da aprendizagem de pares de palavras. Foram oferecidas três alternativas aos participantes para aprenderem a lista de pares de palavras: (a) eles poderiam voltar a estudar o par de palavras logo após ter sido apresentado; (b) eles poderiam voltar a estudar o par de 
palavras logo após ter passado por toda a lista de pares de palavras e (c) eles poderiam decidir não estudar os itens novamente.

Os resultados mostraram que as pessoas utilizaram a estratégia de estudar de forma espaçada quando julgavam que os itens a serem aprendidos eram mais fáceis, mas não quando eram julgados mais difíceis. Son (2004) concluiu que o controle metacognitivo da estratégia de aprendizagem está diretamente relacionado ao monitoramento e conseqüente julgamento que a pessoa faz sobre o conteúdo a ser aprendido.

$\mathrm{Na}$ revisão da literatura, constatou-se que na última década intensificaram-se de forma considerável as pesquisas na área de metacognição. Neste artigo foram relatadas algumas delas, tentando mostrar os domínios específicos pesquisados: leitura, matemática, ciências, resolução de problemas, assim como, diferentes metodologias empregadas: intervenção, entrevistas e julgamento de aprendizagem.

Os exemplos relatados acima se constituem como uma amostra de um número cada vez maior de trabalhos empíricos que tem mostrado que a capacidade de monitorar e auto-regular a própria cognição é um fator importante na aprendizagem. Os resultados destes estudos vêm, também, possibilitando a construção de um corpo de conhecimento explícito e sistemático acerca dos mecanismos cognitivos e metacognitivos envolvidos no desempenho dos alunos. A partir dessas informações, professores e psicólogos cognitivos podem desenvolver situações de aprendizagem que propiciem o surgimento e desenvolvimento de habilidades cognitivas necessárias para dar conta da grande quantidade de informações disponíveis no mundo.

\section{Referências}

Blakey, E., \& Spence, S. (2000). Developing metacognition. Syracuse, NY: ERIC Clearinghouse on Information Resources. Retirado em http://ericae.net/edo/ED327218.htm

Boruchovitch, E. (2001). Algumas estratégias de compreensão da leitura de aluno de ensino fundamental. Psicologia Escolar e Educacional, 5(1), 19-26.

Boruchovitch, E., \& Mercuri, E. (1999). A importância do sublinhar como estratégia de estudo de textos. Revista Tecnologia Educacional, 28, 144, 37-40

Brown, A. (1978). Knowing when, where, and how to remember: A problem of metacognition. In R. Glaser (Ed.), Advances in Instructional Psychology (Vol. 1, p. 77-165). Hillsdale, NJ: Lawrence Erlbaum.

Brown, A. (1997). Transforming schools into communuties of thinking and learning about serious matters. American Psychologis, 52(4), 399-413.

Bruer, J. (1995). Escuelas para pensar: Una ciencia del aprendizaje en el aula. Barcelona, España: Paidós.

Chahon, M. (1999). O uso da metacognição no ensino fundamental de matemática: Uma proposta de intervenção. Arquivos Brasileiros de Psicologia, 51(3), 52-59.

Damasio, A. R. (1996). El error de Descartes. La razón de las emociones. Santiago, Chile: Andres Bello.
Erktin, E. (2004). Teaching thinking for mathematics through the enhancement of metacognitives skills. Research in the Schools, 11, 1, 3-13.

Ertmer, P., \& Newby, T. (1996). The expert learner: Strategies, self-regulated, and reflective. Instructional Sciencem, 24, 1-24.

Flavell, J. (1979). Metacognition and cognitive monitoring. American Psychologist, 34, 906-911.

Flavell, J. (1987). Speculations about the nature and development of metacognition. In F. Weinert \& R. Kluwe (Ed.), Metacognition, motivation, and understanding (p. 21-29). Hillsdale, NJ: Lawrence Erlbaum.

Flavell, J., \& Wellman, H. (1977). Metamemory. In R. V. Kail \& J. W. Hagen (Ed.), Perspective on the development of memory and cognition (p. 3-33). Hillsdale, NJ: Lawrence Erlbaum.

Forest-Pressley, D., \& Waller, T. (1984). Cognition, metacognition and reading. New York: Springer-Verlag New York.

Garner, R. (1987). Metacognition and reading comprehension. Norwood, NJ: Ablex.

Jou, G. I. de. (2001). As habilidades cognitivas na compreensão da leitura: Um processo de intervenção no contexto escolar. Tese de Doutorado não-publicada, Curso de Pós-Graduação em Psicologia do Desenvolvimento, Universidade Federal do Rio Grande do Sul, Porto Alegre, RS.

Klauer, K. (1996). Training of inductive reasoning: A developmental program of higher-order cognitive skills. Paper presented of the Symposium Shaping the Mind through Education, Geneve, Switzerland.

Kimball, D., \& Metcalfe, J. (2003). Delaying judgments of learning affects memory, not metamemory. Memory-andCognition 31(6), 918-929.

Leffa, V. (1996). Aspectos de leitura. Uma perspectiva psicolinguística. Porto Alegre, RS: Sagra.

Marx e Silva, M., Fuhrmeister, A., Brum, A., Costa, F., Rosito, G., Pizutti, L., \& Medeiros, M. et al. (2004). A consciência: Algumas concepções atuais sobre sua natureza, função e base neuroanatômica. Revista Brasileira de Psiquiatria do Rio Grande do Sul, 25(1), 52-64.

Mayor, J., Suengas, A., \& González Marqués, J. (1995). Estrategias metacognitivas: Aprender a aprender y aprender a pensar. Madrid, España: Síntesis.

Mevarech, Z., \& Kramarski, B. (2003). The effects of matecognitive training versus worked-out examples on students' mathematic reasoning. The British Journal of Educational Psychology, 73 (4), 449-471.

Miller, P. (1993). Theories of Developmental Psychology. New York: W. H. Freeman.

Nelson, T., \& Narens, L. (1996). Why investigate Metacognition?. In J. Metcalfe \& A. P. Shimamura (Ed.), Metacognition. Knowing. about knowing (pp. 1-27). Cambridge, MA: MIT Press.

Nelson, T., Narens, L., \& Dunlosky, J. (2004). A revised methodology for research on metamemory: Pre-judgment recall and monitoring (PRAM). Psychological-Methods, 9(1), 53-69.

Oliveira, A., Lima, B., Roberty, B., Rodrigues, D. M., Aires, J., Chahon, M., \& Baldissara, S. G. et al. (2000, julho). Metacognição e a aquisição do conceito de enumerabilidade. Trabalho apresentado no III Congresso de Psicologia do Desenvolvimento, Niterói, RJ

Pressley, M., Goodchild, F., Fleet, J., Zajchowski, R., \& Evans, L. (1989). The challenges of classroom strategy instruction. The Elementary School Journal, 89, 301-342. 
Jou, G. I. de, \& Sperb, T.M. (2006). A Metacognição como Estratégia Reguladora da Aprendizagem.

Ribeiro, C. (2003). Metacognição: Um apoio ao processo de aprendizagem. Psicologia: Reflexão e Critica, 16(1), 109-116.

Rozencwajg, P. (2003). Metacognitive factors in scientific problem-solving strategies. European Journal of Psychology of Education 18(3), 281-294.

Sena de Paula, C. (2000, julho). Metacognição em alfabetização. Pôster apresentado no III Congresso de Psicologia do Desenvolvimento, Niterói, RJ

Seminerio, F. L., Anselme, C. R. S., \& Chahon, M. (1999). Metacognição: Um novo Paradigma. Arquivos Brasileiros de Psicologia, 51(1), 110-126.

Son, L. (2004). Spacing one's study: Evidence for a metacognitive control strategy. Journal of Experimental Psychology: Learning; Memory and Cognition, 30(3), 601-604.

Sternberg, R. (2000). Psicologia cognitiva. Por to Alegre, RS: Artes Médicas.

Stillings, N. A, Weisler, S., Chase, C., Feinstein, M., Garfield, J., \& Rissland, E. (1995). Cognitive Science: An introduction. Cambridge, MA: MIT Press.

Thiede, K. W., \& Anderson, M. C. M. (2003). Summarizing can improve metacomprehension accuracy. Contemporary Educational Psychology, 28(2), 129-160.

Tulving, E. (1996). Foreword. In J. Metcalfe \& A. P. Shimamura (Ed.), Metacognition. Knowing about knowing (p. viii-x). Cambridge, MA: MIT Press.

Vidal-Abarca, E. (1990). Un programa para la enseñanza de la comprensión de ideas principales de textos expositivos. Infancia y Aprendizaje, 49, 53-71.

Vieira, E. (1999). Intervenção psicopedagógica na fase de representação mental em resolução de problemas matemáticos. Tese de Doutorado não-publicada, Pontifícia Universidade Católica do Rio Grande do Sul, Porto Alegre, RS.

Yussen, S. (1985). The growth of reflection in children. Orlando, FL: Academic Press.

Zimmerman, B. J., \& Martinez-Pons, M. (1986). Development of a structured interview for assessing student use of selfregulated learning strategies. American Educational Research Journal, 23, 614-628. 